\title{
LOCALIZATION OF ACTIVE SITES ALONG THE MYELINATED GOLDFISH MAUTHNER AXON: MORPHOLOGICAL AND PHARMACOLOGICAL EVIDENCE FOR SALTATORY CONDUCTION
}

\author{
PAUL G. FUNCH, ${ }^{2}$ MALCOLM R. WOOD, AND DONALD S. FABER \\ Division of Neurobiology, Department of Physiology, State University of New York, Buffalo, New York 14214
}

Received November 29, 1983; Revised April 23, 1984; Accepted May 2, 1984

\begin{abstract}
Injections of Lucifer Yellow (LY) and horseradish peroxidase (HRP) were made within the myelin sheath of the goldfish Mauthner axon to determine the domains of individual oligodendrocytes. Long segments of the myelin sheath were stained with both markers. The lengths and locations of these sheath segments were analyzed in whole mount preparations (LY) or in reconstructions of serial vibratome sections (HRP). The termination sites of individual myelin sheaths, relative to gross anatomical landmarks of the brain, were consistent within and between all fish studied. In particular, the average locations of the termination sites were separated by 2.2 to $2.6 \mathrm{~mm}$ and corresponded to the brain regions where active site foci have been previously localized electrophysiologically. Individual sheath segments generally spanned the entire distance between adjacent active sites. The node-internode-node structure of the Mauthner axon that is suggested by these findings was further tested by ejecting tetrodotoxin (TTX) at various discrete rostral-caudal locations just outside the fiber. Large all-or-nothing components of the antidromic action potential were rapidly blocked (within seconds) only when the TTX ejections were made within a few hundred micrometers of the active site foci. The amplitudes of these blocked components are also consistent with predictions based upon previous electrophysiological analyses which demonstrated an active site spacing of 2.2 to $2.6 \mathrm{~mm}$, a space constant of $5.0 \mathrm{~mm}$, and a safety factor of 6 for impulse propagation. It is concluded from these morphological, pharmacological, and electrophysiological observations that the Mauthner axon possesses nodes separated by 2.2 to $2.6 \mathrm{~mm}$ and that a single oligodendrocyte spans an internodal region. Although nodal ultrastructure remains to be described, these results rule out the possibility that each of the short $(\sim 50 \mu \mathrm{m})$, closely spaced (average separation $=155 \mu \mathrm{m}$ ) axon collaterals is a site of action current production.
\end{abstract}

The morphological substratum of saltatory conduction along vertebrate myelinated axons is the node of Ranvier. This gap between successive myelinating glial cells provides a low resistance pathway from axoplasm to extracellular space, and the nodal axolemma contains a high density of voltage-sensitive sodium channels which supports electrogenesis. However, the Mauthner axon of fish is distinguished by reports that it lacks typical nodal gaps (Graham and O'Leary, 1941; Leghissa, 1956; Gray, 1970; Celio, 1976; Koenig, 1978; Yasargil et al., 1982). The only openings in the Mauthner axon's myelin sheath that have been observed are the tips of its relatively short $(\sim 50 \mu \mathrm{m})$ and closely spaced (100 to $300 \mu \mathrm{m})$ collaterals which make synaptic contact with interneurons and motoncurons (Barets, 1961; Bartelmez, 1915; Coghill, 1934; Bodian, 1952; Leghissa, 1956; Retzlaff and Fontaine, 1960; Diamond et al., 1970; Gray, 1970; Diamond, 1971; Celio et al., 1979; Funch and Faber, 1982b; Yasargil et al., 1982). These morphological observations have led to the hypothesis that individual collateral tips are "nodal equivalents" which produce the action currents necessary for impulse propagation (Celio et al., 1979; Greeff, 1976,

\footnotetext{
${ }^{1}$ This work was supported in part by National Institutes of Health Grant NS-17063. We thank J. Lakatos for photographic assistance and J. Seiler for secretarial help.

${ }^{2}$ To whom correspondence should be addressed.
}

1977). Greeff and Yasargil (1980) offered electrophysiological evidence in support of that hypothesis with their analysis of longitudinal action currents recorded along the surface of the spinal cord. In contrast, the electrophysiological investigations of Funch and Faber (1982b) ruled out the possibility that each axon collateral contributes equally to action current production since the active site foci were found to be spaced at intervals of 2.0 to $2.8 \mathrm{~mm}$, or about 10 times the average intercollateral separation. Nevertheless, Yasargil et al. (1982) have recently used several histological staining protocols together with EM analysis to demonstrate that the unmyelinated collateral tips have morphological properties in common with nodal membranc regions. These interesting data have reasserted the collateral hypothesis and posed these associated questions: What is the domain of a single oligodendrocyte which myelinates the Mauthner axon? Does it span only the region between adjacent collaterals, or does it ensheath some other length of the Mauthner axon that is unrelated to individual collaterals? Thus, the experiments described here were designed both to obtain additional evidence to distinguish between the two hypotheses of saltatory conduction in the Mauthner axon and to contribute to our understanding of vertebrate CNS axon-oligodendrocyte structural relationships. Some of these results have been pub. lished in abstract form (Funch and Faber, 1983; Wood et al., 1983). 


\section{Materials and Methods}

Experimental animals and surgical procedures. Common goldfish, Carassius auratus, with body lengths of 7.5 to $12.5 \mathrm{~cm}$ were used in these experiments. They were initially anesthetized with tricaine melhanesulfonate (Finquel, Ayerst; $0.1 \mathrm{gm} / \mathrm{liter}$ ) and then immobilized with $d$-tubocurarine $(0.1$ to $0.3 \mathrm{ml}, 3 \mathrm{mg} /$ liter; equivalent to 1 to $3 \mu \mathrm{g} / \mathrm{gm}$ of body weight, i.m.). 'They were held rigidly in a recording chamber by stainless steel rods and respired and continuously anesthetized with tricaine methanesulfonate in aerated dechlorinated tap water which was pumped through the mouth and over the gills. The medulla and the rostral few millimeters of the spinal cord were exposed, and the meningeal membrane was dissected apart to facilitate microelectrode penetrations. The spinal vertebrae were exposed near the caudal base of the dorsal fin, a distance of nearly one-half the body length from the caudal border of the vagal lobes, and a bipolar electrode contacting the undissected vertebral column was used to activate antidromically the Mauthner axon and other fibers in the spinal cord.

Electrophysiological and dye injection techniques. Intracellular recordings from the Mauthner axon were obtained with glass micropipettes (American Glass Co., outer diameter $2 \mathrm{~mm}, 1.2 \mathrm{~mm}$ inner diameter, capillary fiber) filled with $2.7 \mathrm{M} \mathrm{KCl}$ (initial resistances, 5 to $10 \mathrm{megohms}$ ). These impalements were used to identify the axon positively as well as to monitor action potential parameters to ensure that no damage resulted from the dye injections. For dye injections, the tips of the micropipettes were first backfilled by capillary action for several minutes with a solution of the dye, either Lucifer Yellow (LY; Sigma Chemical Co., $0.4 \mathrm{gm} /$ liter in $1 \mathrm{M} \mathrm{LiCl}$ ) or horseradish peroxidase (HRP; Sigma, type VI, $0.4 \mathrm{gm} /$ liter in $0.1 \mathrm{M} \mathrm{KCl}$ plus 0.025 $\mathrm{M}$ Tris buffer, $\mathrm{pH} 7.6$ ), and then the microelectrode barrel was filled with the appropriate solution without any dye. LY electrode tips were broken to yield initial resistances of 10 to 30 megohms. Broken electrodes filled with HRP were beveled (Sutter Instrument Co.) to initial resistances of 10 to 30 megohms. Dyes were pressure injected (20 psi) with 1 - to 4 -sec pulses that were repeated 5 to 10 times at a rate of about one per minute. The general impression was that the injections into the myelin were more likely to be successful when the action potential recorded through the dye-containing electrode was reduced by more than half immediately after the pulse was begun and then recovered to its original amplitude over a period of 10 to $20 \mathrm{sec}$ after the pulse was terminated.

Morphological techniques. For fish with cells that had been injected with LY, whole mount preparations of the brain and spinal cord were prepared as follows. The fish were removed from the recording chamber within 0.5 to $3 \mathrm{hr}$ after the injection and perfused through the conus arteriosus with 30 to $75 \mathrm{ml}$ of cold $10 \%$ buffered formalin phosphate for 20 to $30 \mathrm{~min}$. Then the spinal column, cranium, and surrounding tissues were dissected apart from the body of the fish and immersed in fixative for several hours at $4^{\circ} \mathrm{C}$. The brain and rostral centimeter of spinal cord were dissected free, washed in cold fixative, and held down with fine wire pins in a Petri dish whose bottom had been coated with Sylgard. They were then dehydrated in a graded series of ethanol solutions $(50 \%, 70 \%, 90 \%$, and $100 \%$, twice for $20 \mathrm{~min}$ each) and finally cleared in methyl salicylate (oil of wintergreen, $n_{D}=1.536$ ). The whole brains were examined using fluorescence microscopy (Stewart, 1978) and photographed using Ektachrome 400 or Tri-X film.

Following pressure injections of HRP, fish were kept in the recording chamber for 3 to $6 \mathrm{hr}$ to allow for sufficient diffusion of the HRP. The fish were then perfused with glutaraldehyde $(2.5 \%)$ in sodium cacodylate $(0.1 \mathrm{M}, \mathrm{pH} 7.2)$. The brain and proximal region of the spinal cord were fully exposed dorsally and immersed in situ in the same fixative for $1 \mathrm{hr}$. The tissue was dissected out and fixed an additional $2 \mathrm{hr}$, washed in phosphate buffer $(0.15 \mathrm{M})$ for $1 \mathrm{hr}$, and placed in gelatin/ albumin mixture overnight at $4^{\circ} \mathrm{C}$. Treatment continued as follows. The brain was trimmed anteriorly by removing the tectal lobes; the remaining brain tissue and attached length of spinal cord $(\sim 0.5 \mathrm{~cm})$ were embedded in a mixture of four parts gelatin/albumin to one part $5 \%$ glutaraldehyde (modified from Frank et al., 1980); 30- $\mu \mathrm{m}$ Vibratome (Lancers) sections were cut horizontally and free floated in a compartmentalized staining net in a Petri dish containing phosphate buffer $(0.15 \mathrm{M})$. To visualize the HRP, sections were treated with ice-cooled diaminobenzidine (DAB; Adams, 1981) for $1 \mathrm{hr}$ with continuous agitation, followed by the addition of $\mathrm{H}_{2} \mathrm{O}_{2}(3 \%)$ for an additional $20 \mathrm{~min}$. The sections were washed in phosphate buffer, serially mounted on subbed slides, and air dried. Subsequently, the sections were dipped briefly in phosphate buffer, counterstained in neutral red for $5 \mathrm{~min}$, rapidly dehydrated, cleared, and permanently embedded in Permount.
Pharmacological techniques. For pharmacological experiments, the tip of a microelectrode was backfilled with a solution of TTX (Sigma; $30 \mu \mathrm{M}$ ) and the dye fast green (Sigma; $20 \mathrm{mg} / \mathrm{ml}$ ) in $0.2 \mathrm{M} \mathrm{NaCl}$ in 20 mM HEPES buffer ( $\mathrm{pH} 7.2$ ). The microelectrode barrel was then filled with $0.2 \mathrm{M} \mathrm{NaCl}$ in HEPES buffer solution. The myelin sheath of the Mauthner axon was located using the electrophysiological criteria described below, and the TTX electrode was positioned 5 to $10 \mu \mathrm{m}$ outside the sheath. The TTX solution was pressure ejected, under visual control, with a pulse duration sufficient to eject a roughly 100 $\mu \mathrm{m}$ diameter sphere of TTX solution.

Orthodromic activation of the Mauthner cell was accomplished by stimulating the posterior branch of the eighth nerve with a bipolar electrode at the lateral margin of the medulla. This nerve branch contains vestibular and auditory afferents which monosynaptically excite the Mauthner cell's lateral dendrite.

\section{Results}

\section{E'lectrophysiological recordings from within the myelin sheath}

When the myelin sheath of a Mauthner axon was just penetrated with a glass microelectrode (shown schematically in Fig. $1 B$ ) a variable DC potential was recorded. Although it was generally negative relative to a distant extracellular reference electrode, as in Figure $1 A(0 \mu \mathrm{m}$ trace $)$, this was not always so. With further gradual penetration into the myelin sheath of from 10 to $20 \mu \mathrm{m}$ (Fig. $1 A, 5$ to $18 \mu \mathrm{m}$ traces), the DC potential did not vary in any consistent way and remained within a range of about -20 to $+20 \mathrm{mV}$. Thus, while such recordings were stable for many tens of minutes, they cannot be considered as being entirely from within the intracellular milieu of the oligodendrocyte. Finally, with an additional slight advance of the microelectrode, a very large negative potential of typically -85 $\mathrm{mV}$ was suddenly recorded (Fig. $1 A, 23 \mu \mathrm{m}$ trace), indicating that the axolemma had been penetrated. The amplitude of the Mauthner axon's action potential, recorded extra-axonally, steadily increased as the microelectrode advanced through the sheath. This is the expected result if the electrode is recording across a greater and greater fraction of a passive resistance existing between the interior of the axon and the distant external reference electrode. The variable DC potentials simultaneously recorded could be expected to be due to a combination of tip potential artifacts produced as the microelectrode pushes through the fatty myelin sheath plus occasional "damaged cell" recordings from portions of cytoplasmic channels. Just before penetration of the axolemma occurred, the action potential amplitude was at least $50 \mathrm{mV}$, and, as shown in Figure $1 A$ (18 $\mu \mathrm{m}$ trace), it could be as much as $80 \mathrm{mV}$. By comparison, the intra-axonal action potential amplitude was typically 115 to $125 \mathrm{mV}$.

\section{Determinations of oligodendrocytic sheath domains}

Intrasheath LY injections. To inject dyes into the myelin sheath, a dye-filled microelectrode was advanced into the sheath while an antidromic action potential was elicited. When the recorded action potential amplitude was 30 to $50 \mathrm{mV}$ (and little or no DC potential was present), the dye was pressure ejected from the electrode. In no experiment was the axon stained unless intra-axonal penetration, as deduced from the sudden appearance of a large $(-85 \mathrm{mV})$ resting potential, was followed by application of pressure to the microelectrode. Similarly, the sheath was never stained if only the axon was injected with dye. Even with long periods of stimulation at high frequency, there was no transfer of LY either from the sheath to the axon or vice versa, which suggests that there are no voltagesensitive pathways for LY diffusion between the two cell types. In all experiments, intracellular staining was readily distinguished from the infrequent instances of extracellular dye leakage.

A single injection of LY performed as described above resulted in the selective staining of long segments of the sheath 


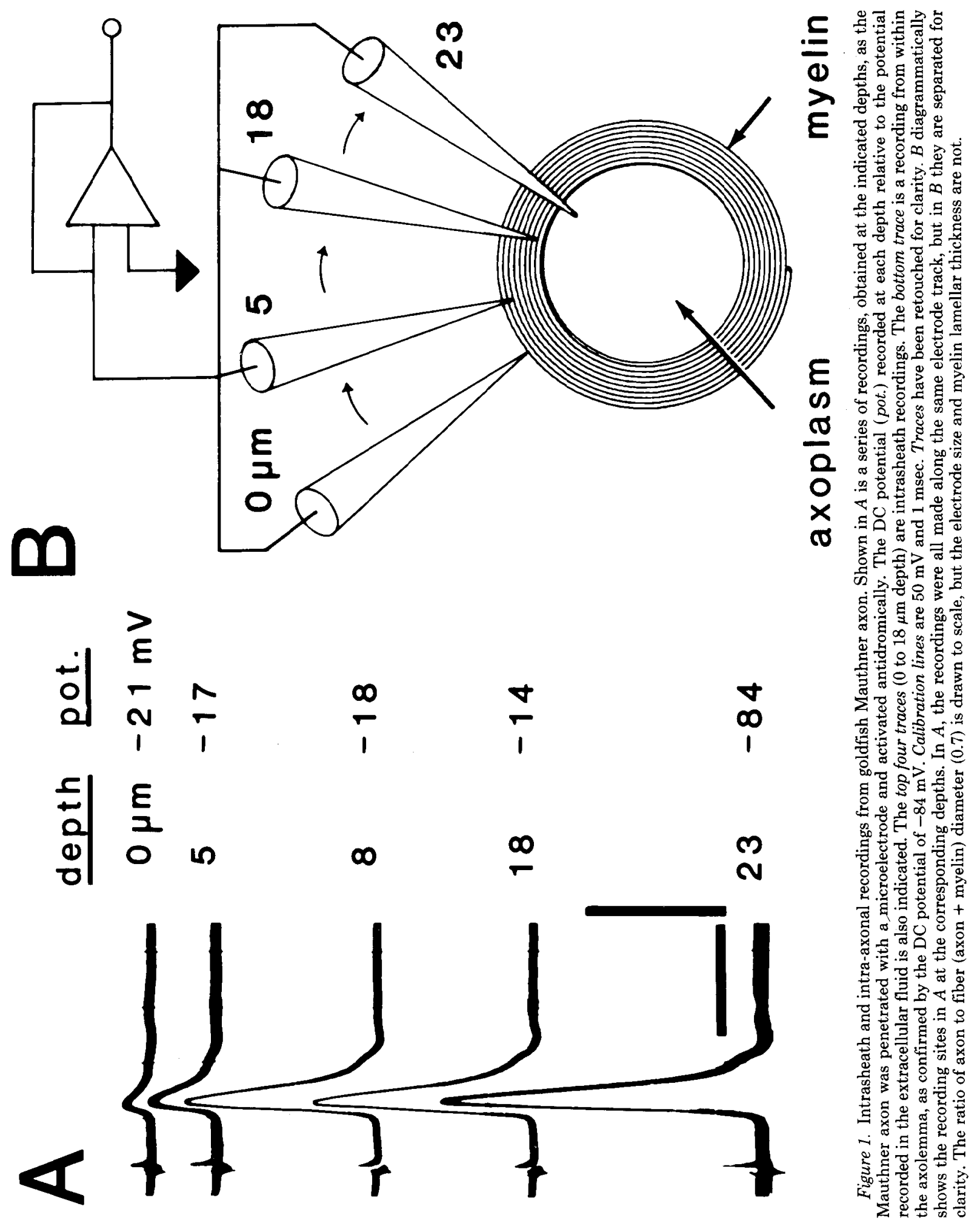


which terminated nonrandomly along the longitudinal axis of the brain and spinal cord. Furthermore, the density of the staining along the axis of the axon was typically asymmetric about the injection site. To assess the pattern of such staining from fish to fish, the most rostral and most caudal "points" to which the dye diffused were measured relative to a readily identifiable gross anatomical landmark, namely, the posterior margin of the vagal lobes. When both the axon and its sheath were injected, the termination "points" generally had a well defined, tapered appearance. However, when only the myelin sheath was stained, the termination "points" were occasionally difficult to localize precisely, as demonstrated in Figure $2 A$, which shows the most indistinct termination encountered. The more distinct "point" of termination shown in Figure $2 B$ was observed in most experiments, although clearly even such a case as this required a subjective decision for its localization. Nonetheless, the uncertainty in the precise "point" of termination was always much less than the total length of myelin sheath stained.

Figure 3 gives a summary of all myelin sheaths stained with LY and analyzed as described above. In addition to showing the total length of myelin sheath which was stained, the sites where dye injections were made are also indicated with arrowheads.

The salient observations from Figure 3 are as follows. (1) Long lengths of the myelin sheath were stained with single dye injections. The two shortest stained lengths were $1.31 \mathrm{~mm}$ (experiment $4 R$ ) and $2.16 \mathrm{~mm}$ (experiments $14 R$ and $14 L$ ), and the longest was $5.52 \mathrm{~mm}$ (experiments $25 R$ and $25 L$ ). (2) Terminations of the stained myelin sheaths were nonrandomly distributed (see the histogram) and, in fact, occurred in discrete regions of the medulla and spinal cord. (3) Regardless of the rostral-caudal level of an injection site, single injections of LY stained whole segments of myelin sheath between the average termination sites (vertical dotted lines). (4) When the sheaths of both Mauthner axons were stained in a single fish, they terminated at the same rostral-caudal level. This was true both when the same segment was stained (cf. left $(L)$ and right $(R)$ for experiments 14,19 to 22 , and 24 to 26 ) and when consecutive segments were stained (cf. experiments $1 L$ and $1 R$ ). (5) LY could apparently pass across termination sites (dotted vertical lines) to stain very long lengths of the sheath $(4.13$ to $5.52 \mathrm{~mm})$, as in those experiments grouped below the thick horizontal dashed line; two such cases are shown in Figure 4.

In addition to the experiments summarized in Figure 3, there were two experiments in which intrasheath injections were made 5.1 to $5.5 \mathrm{~mm}$ posterior to the vagal lobes. In one experiment, the myelin sheath stained was $2.14 \mathrm{~mm}$ long and in the other, $4.32 \mathrm{~mm}$; both sheaths extended only caudally from the injection sites. Presumably, all of the longest myelin sheaths stained represent dye coupling between two adjacent segments. Such coupling was observed at all levels of the neuraxis which we studied. It should be noted that even when very long segments of the myelin sheath were stained, the rostral and caudal sites of termination were very similar from experiment to experiment, despite widely varying sites of LY injection.

Intrasheath HRP injections. As indicated above, the occasional extensive staining of a myelin sheath with LY (e.g., Fig. 4) suggested the possibility that adjacent segments of the Mauthner axon's myelin sheath might be dye coupled to each other. If this were so, then even the shortest myelin sheath segments observed could have been the result of LY dye coupling between several shorter sheath cells. To investigate this possibility, experiments were performed using intrasheath injections of HRP. HRP was successfully injected into a total of 19 sheaths in 10 of 16 fish. The time period apparently necessary for diffusion of the HRP throughout the sheath (several hours) was generally much longer than that required for adequate LY staining (less than $0.5 \mathrm{hr}$ ). Due to its high molecular weight, HRP does not pass from one cell to another, with only rare exceptions. Figure 5 gives a summary of these experiments in a manner identical to that employed in Figure 3.

The data in Figure 5 demonstrate a number of important points. First, long lengths of the myelin sheath were stained with single HRP injections (experiments grouped above the horizontal dashed line). The two shortest sheath segments stained were $0.70 \mathrm{~mm}$ (experiment $88 R$ ) and $0.85 \mathrm{~mm}$ long (experiment $85 R$ ), and the longest was $2.47 \mathrm{~mm}$ (experiment $81 R$ ). Second, the terminations of the myelin sheath segments were clustered (see the histogram) in the same discrete regions of the brain and spinal cord where the average termination sites of LY staining occurred. Third, regardless of where the injection was made, single injections of HRP could stain the whole segment of myelin between average termination sites (vertical dotted lines). In several experiments, the staining pattern was quite asymmetric with respect to the injection site, suggesting that a distinct diffusion barrier exists at the termination sites.

As noted above, in some cases the extent of HRP diffusion was relatively short, i.e., less than $1 \mathrm{~mm}$. To test whether such

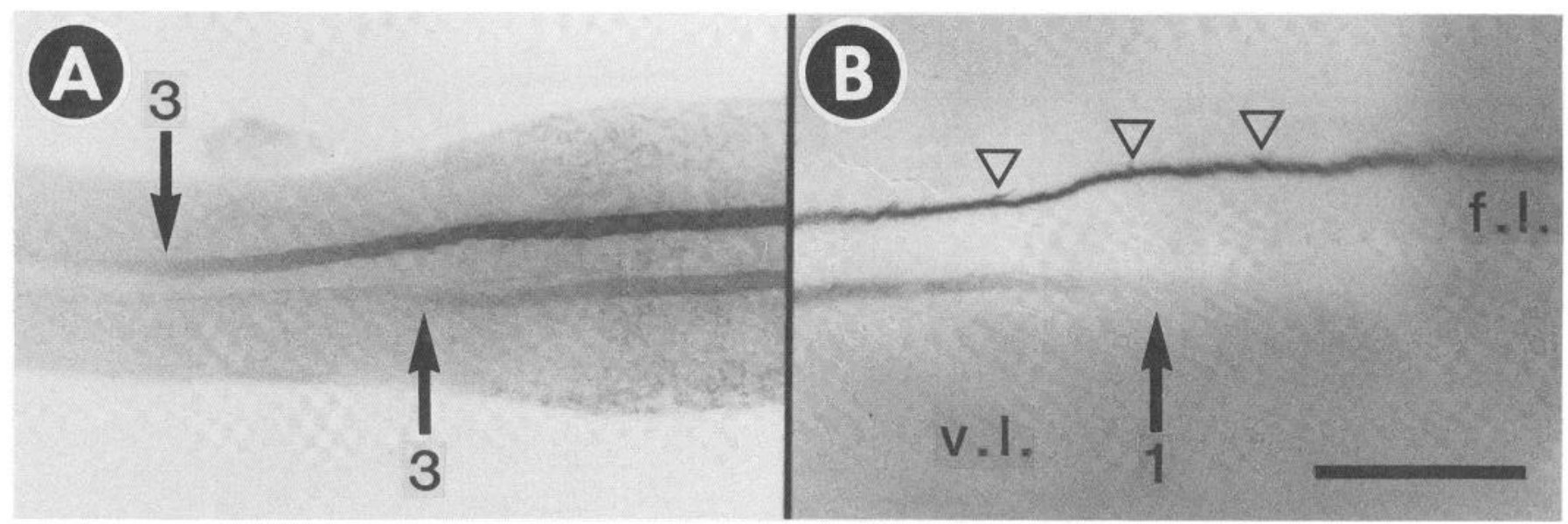

Figure 2. Terminations of staining of Mauthner axon myelin sheaths injected with LY. $A$ and $B$ are negative photographs of cleared whole mount preparations. In $A$ (experiment 19), the left and right sheaths terminate indistinctly at region 3 (see Fig. 3 ). The arrows indicate the points of termination used for the graphs in Figure 3. In $B$ (experiment 6), the myelin sheath of the right Mauthner axon is shown terminating more distinctly, at region 1 . The left Mauthner axon was also injected, and three short collaterals $(\nabla)$ were visible (the right one is not in focus in this photograph). f.1., facial lobe; v.1., vagal lobe. Rostral is to the right, and the calibration line is $500 \mu \mathrm{m}$ in $A$ and $B$. 
results indicated the existence of short sheath segments or simply a difficulty of HRP diffusion throughout the entire segment, multiple HRP injections were made over regions that were bounded by average sites of termination defined in Figure 3 . These experiments, grouped below the dashed line, show that longer segments could be stained with multiple injections and that the terminations of the longer sheath segments still occurred in the same regions of the medulla and spinal cord as determined previously. Finally, when the sheaths of both Mauthner axons were stained, they terminated at the same rostral-caudal level. As in the LY experiments, this was so both when the same segment was stained (cf. $L$ and $R$ for experiments 83,84 , and 91 ) and when consecutive segments were stained (cf. $L$ and $R$ for experiments $81,85,89$, and 90 ).

A summary of the total extents of myelin sheath stained by both LY (single injections) and HRP (all experiments) is shown in Figure 6. Whereas the LY-stained segments display a bimodal distribution of lengths, consistent with dye coupling, the HRP-injected segments display a unimodal distribution whose peak corresponds with the first peak of the LY distribution.

\section{Distribution of Mauthner axon collaterals}

In several of the HRP experiments, the Mauthner axon was injected, either alone or in addition to its myelin sheath. It was discovered that with the protocol used for tissue preparation, there was excellent preservation of the axon collaterals. Several collaterals stained with HRP reaction product are shown in Figure 7. It should be noted that the collaterals are often quite close to each other (Fig. 7A) and that multiple collaterals may arise from the same site (Fig. $7 B$ ).

With such unambiguous views of the collaterals in longitudinal sections, in contrast to the capricious collateral staining we obtained with LY (see Fig. $2 B$ and also Fig. 1 in Yasargil et al., 1982), we reinvestigated the spatial distribution of the Mauthner axon collaterals in the rostral portion of spinal cord and medulla. A summary of the intercollateral separations we observed in eight fish is shown in Figure 8. Their average separation was $155 \mu \mathrm{m}$, although there was no apparent regularity in their spacing. Of particular interest were the many separations of $<100 \mu \mathrm{m}$ (e.g., Fig. $7 A$ ), and especially striking were the paired and even triplet (e.g., Fig. $7 B$ ) collaterals which emerged from the Mauthner axon. Relatively few such multiple collaterals were observed, and they did not occur at identifiable locations along the Mauthner axons.

\section{Spatial sensitivity of the Mauthner axon to localized extracellular ejections of TTX}

When ejections of small amounts of TTX were made just outside the myelin sheath of the Mauthner axon (see "Materials and Methods") in rostral-caudal locations further than several hundred micrometers from the average termination sites determined from the LY and HRP experiments, they had either no effect or a delayed one (several minutes). In contrast, when TTX was ejected within a few hundred micrometers of those average termination sites, there was a rapid (within a few seconds) blockage of a large all-or-nothing action potential component and a corresponding decrement in the maximal rate of rise of the impulse (Fig. 9).

As many as three consecutive action potential components could be blocked by ejections made $1.4 \mathrm{~mm}$ rostral, $1.2 \mathrm{~mm}$ caudal, and $3.2 \mathrm{~mm}$ caudal to the posterior margin of the vagal lobes, respectively, and yet the antidromic action potential could still just invade the axon hillock-initial segment region (Fig. 9, trace 3). As many as four consecutive action potential components could be blocked (the fourth ejection being made $5.5 \mathrm{~mm}$ caudal to the vagal lobes), and yet the orthodromic action current was still just sufficient to activate the next (fifth) downstream active site. Table I summarizes data from eight experiments where the all-or-nothing losses in antidromic action potential amplitude and its maximal rate of rise were recorded after successful TTX ejections. These ejections were made consecutively at each of the sites listed above. The stepwise decrements were comparable to those predicted from a simple cable model of the $\mathrm{M}$-axon (Funch and Faber, 1982a) and its known electrophysiological properties (Funch and $\mathbf{F a}$ ber, 1982b).

\section{Discussion}

Correlation of myelin sheath staining with electrophysiological data. From our results it appears that intrasheath injections of LY and HRP apparently selectively stain the myelinating oligodendrocytes. As shown in Figure 5, single injections of HRP stained myelin sheath segments up to $2.5 \mathrm{~mm}$ in length. The oligodendrocyte which produces the myelin sheath surrounding the Mauthner axon must be as large as that since HRP is not expected to pass from one cell to another over the time course of these acute experiments. Furthermore, the average sites of termination of the myelin sheath segments stained with either LY or HRP, as shown in Figures 3 and 5 , were clustered; virtually no overlap occurred between clusters despite a wide distribution of injection sites. The overall average sites of termination for both the LY and HRP experiments combined are $3.8 \mathrm{~mm}$ rostral, $1.4 \mathrm{~mm}$ rostral, $1.2 \mathrm{~mm}$ caudal, and $3.4 \mathrm{~mm}$ caudal to the posterior margin of the vagal lobes, which yield distances of $2.4 \mathrm{~mm}, 2.6 \mathrm{~mm}$, and $2.2 \mathrm{~mm}$ between the termination sites. Thus, the Mauthner axon's oligodendrocytes, which may be 2.2 to $2.6 \mathrm{~mm}$ in length, are arranged in fixed positions relative to gross anatomical landmarks of the goldfish brain. In particular, the first oligodendrocyte begins either at the level of the decussation or at the initial segment of the Mauthner axon in the rostral portion of the medulla. Additional HRP experiments are necessary to assess the distal limit of the oligodendrocyte which terminates proximally at the initial segment. The second oligodendrocyte begins roughly $2.4 \mathrm{~mm}$ more caudally, just posterior to the facial lobe. The third and fourth are $1.2 \mathrm{~mm}$ and $3.4 \mathrm{~mm}$ caudal to the posterior margin of the vagal lobes, respectively. While only two experiments provided data on sheath segments in the rostral portion of the spinal cord, these also indicated lengths of about $2.2 \mathrm{~mm}$. Interestingly, this distance is the same as spinal segment length in these fish. It is not known whether this coincidence is associated with any specific synaptic relationships which may have an inhomogeneous distribution along the length of the spinal cord.

However, the most striking correlations are that the average sites of termination of the oligodendrocytes have the same separations and the same absolute locations as the active sites which were found using electrophysiological techniques (Funch and Faber, 1982b). The only lack of correspondence may be in the region of the decussation of the Mauthner axons. Electrophysiological analysis lacks the necessary resolution to determine whether separate active sites exist there and in the axon hillock-initial segment region, and, as noted above, the morphological data are inconclusive as to whether there is a short $(\sim 300 \mu \mathrm{m})$ oligodendrocyte segment between the two regions. It should be emphasized that these correlations are based upon the results of two independent studies using different fish. It is important to consider the errors introduced by this experimental design. The fish were of the same size range $(7.0$ to $12.5 \mathrm{~cm}$ body length) in both studies, but the variability in fish size is certainly expected to contribute to variability in the internodal lengths which were measured (Thomas and Young, 1949), even though elongation of the medulla and rostral portion of the spinal cord is not directly proportional to the increase in body 


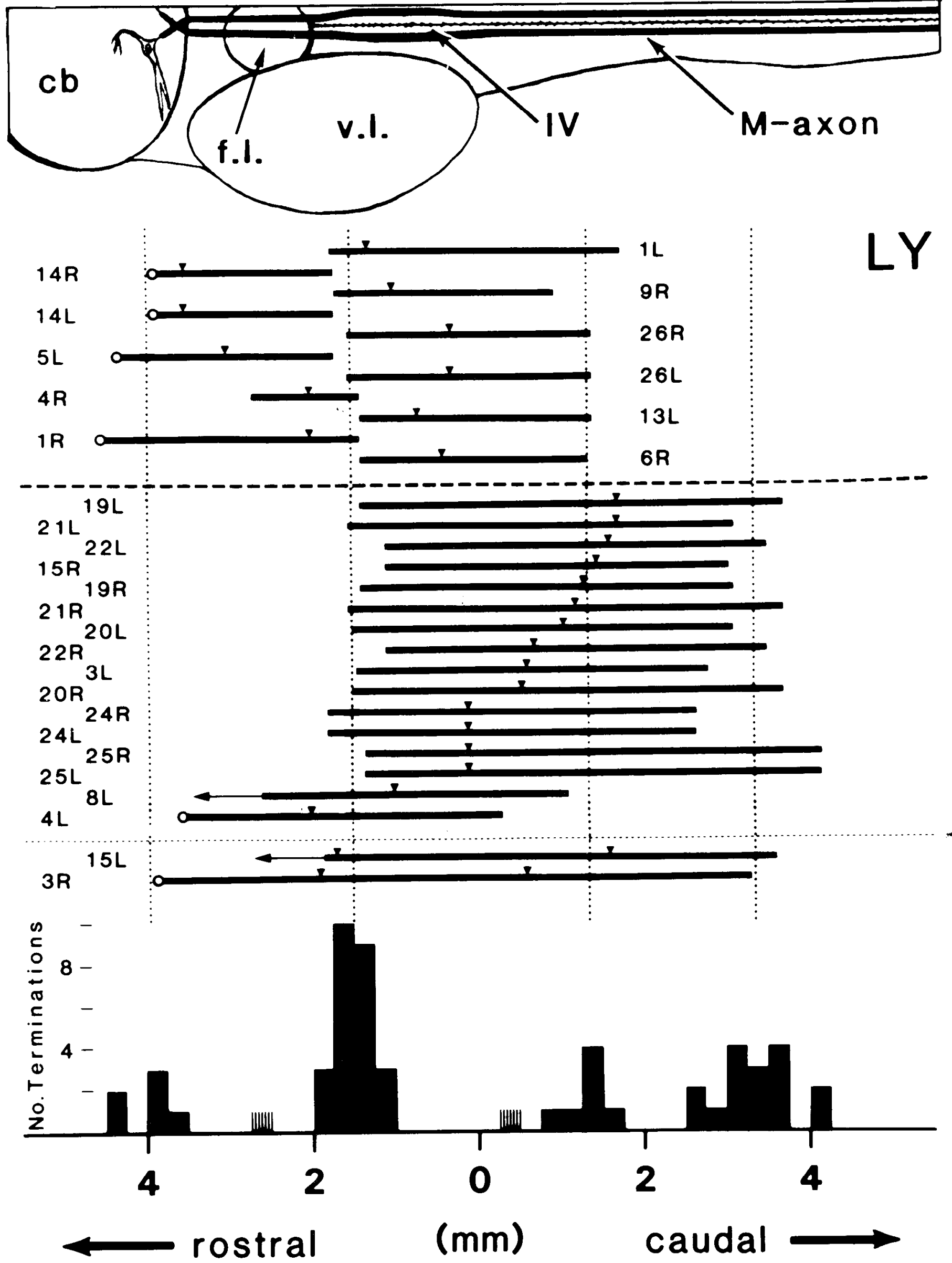




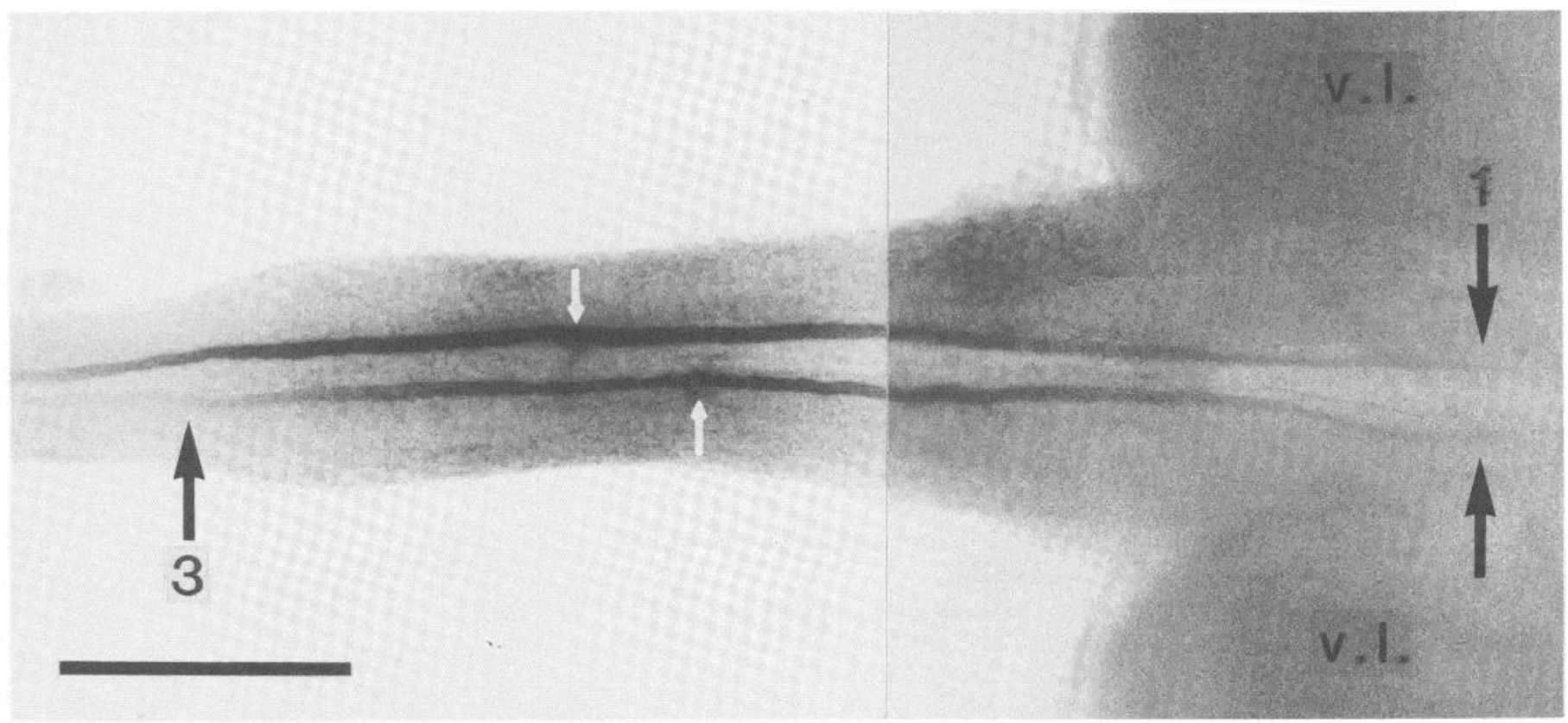

Figure 4. Possible LY dye coupling between adjacent internodal myelin sheaths of goldfish Mauthner axons. Negative photographic montage of cleared whole mount preparation (experiment 19). For each axon, a single intrasheath injection was made at the site indicated by the white arrow. The left and right sheaths terminate at somewhat different caudal levels (region 3; left sheath termination is just beyond the lefthand edge of the photograph), but at the same rostral level (region 1); their lengths are 5.1 and $4.5 \mathrm{~mm}$, respectively. The righthand edge of this figure corresponds to the caudal margin of the facial lobe. v.1., vagal lobe. Calibration line is $1.0 \mathrm{~mm}$.

length. Nevertheless, there was no correlation between length of sheath segments stained and fish size for either LY or HRP experiments. Such a relationship was likely obscured by several other errors which were involved in all of the measurements of myelin sheath segment lengths and locations. First, as shown in Figure 2, the subjective assignment of a "point" to the termination of a sheath segment has a possible error of as much as several hundred micrometers. Second, by using the posterior margin of the vagal lobes as a reference point, we took advantage of the only "fixed," centrally located landmark that could be seen both in LY whole mounts and HRP vibratome sections. However, the shape of the vagal lobe's posterior margin was clearly variable from fish to fish; in fact, the left and right vagal lobes did not always end at the same rostral-caudal level in a single fish. Furthermore, since the vagal lobes overhang the medulla, their posterior margin is more caudal when viewed in situ or in a whole mount preparation than when seen in a vibratome section at the level of the Mauthner axons. When measurements were made from sections, the section with the most caudal portion of the vagal lobes was superimposed to obtain a more accurate determination of relative location. Nevertheless, these orientation difficulties could contribute an error of perhaps several hundred micrometers to the location of the termination "points." Third, LY could apparently pass from one oligodendrocyte to an adjacent one. Thus, there is no certainty that any particular termination site represented a cellular border. However, the clearly bimodal distribution of sheath lengths stained by LY (Fig. 5) suggests that a barrier with "all-or-nothing" properties may exist between oligodendrocytes. That barrier either prevents LY from passing to the next cell or else it essentially allows LY to pass without impediment into the adjacent cell. It is not clear what conditions predispose the apparent gating of this diffusion barrier. While the bimodal distribution suggests that LY staining tends to stop at cellular borders, nevertheless, LY diffusion across cellular borders may account for some portion of the observed variability in sheath lengths.

The situation with the HRP injections was somewhat different. Clearly HRP diffused more slowly through the fine cytoplasmic channels than did LY, and, in several of the single injection experiments (Fig. 5, above the dashed line), it was our impression that sheath terminations were determined by a diffusional limitation rather than by a cellular border. This notion was supported by the results of experiments in which two or three widely spaced injections were made into the myelin sheath. These results (grouped below the horizontal dashed line in Fig. 5) showed that longer, but also more uniform, lengths of the myelin sheath were stained. These longer segments corresponded with the longest segments we obtained with single injections of HRP, so we conclude that the multiple injections were made within single cellular elements. Thus, one end of three of the shortest sheath segments stained (lined boxes in

Figure 3. Summary of Mauthner axon myelin sheath segments stained with intrasheath LY injections. At the top is a scale drawing of the goldfish brain with the left Mauthner cell and both axons superimposed. $c b$, cerebellum; f.1., facial lobe; v.1., vagal lobe; $I V$, fourth ventricle. Below, and drawn to the same scale, are total lengths of individually stained myelin sheaths (black bars). Intrasheath injection sites are indicated with arrowheads. Terminations at the initial segment are labeled with open circles. $L$ and $R$ refer to left and right Mauthner axons. Left-pointing arrows are sheaths whose rostral terminations could not be seen clearly through the facial lobe. The thick horizontal dashed line separates those experiments in which a single LY injection resulted in the staining of either a short segment, $<3.5 \mathrm{~mm}$ (above), or a long segment, $>4.1 \mathrm{~mm}$ (below). Below the thin horizontal dashed line are experiments where two intrasheath injections were made. At the bottom is a histogram of sheath termination sites, showing clusters at separations of 2.0 to $2.8 \mathrm{~mm}$. The vertical dotted lines indicate average termination sites which were obtained from the data in the shaded histograms and are, from left to right: $4.0 \mathrm{~mm}$ rostral, $1.5 \mathrm{~mm}$ rostral, $1.3 \mathrm{~mm}$ caudal, and $3.3 \mathrm{~mm}$ caudal to the posterior margin of the vagal lobe $(0 \mathrm{~mm})$; these correspond to the initial segment and nodal regions 1,2 , and 3 , respectively. Two terminations (lined boxes in the histogram) were not included in averages because they were farther than 2 SD from the means and probably represented sheaths that were incompletely stained. 


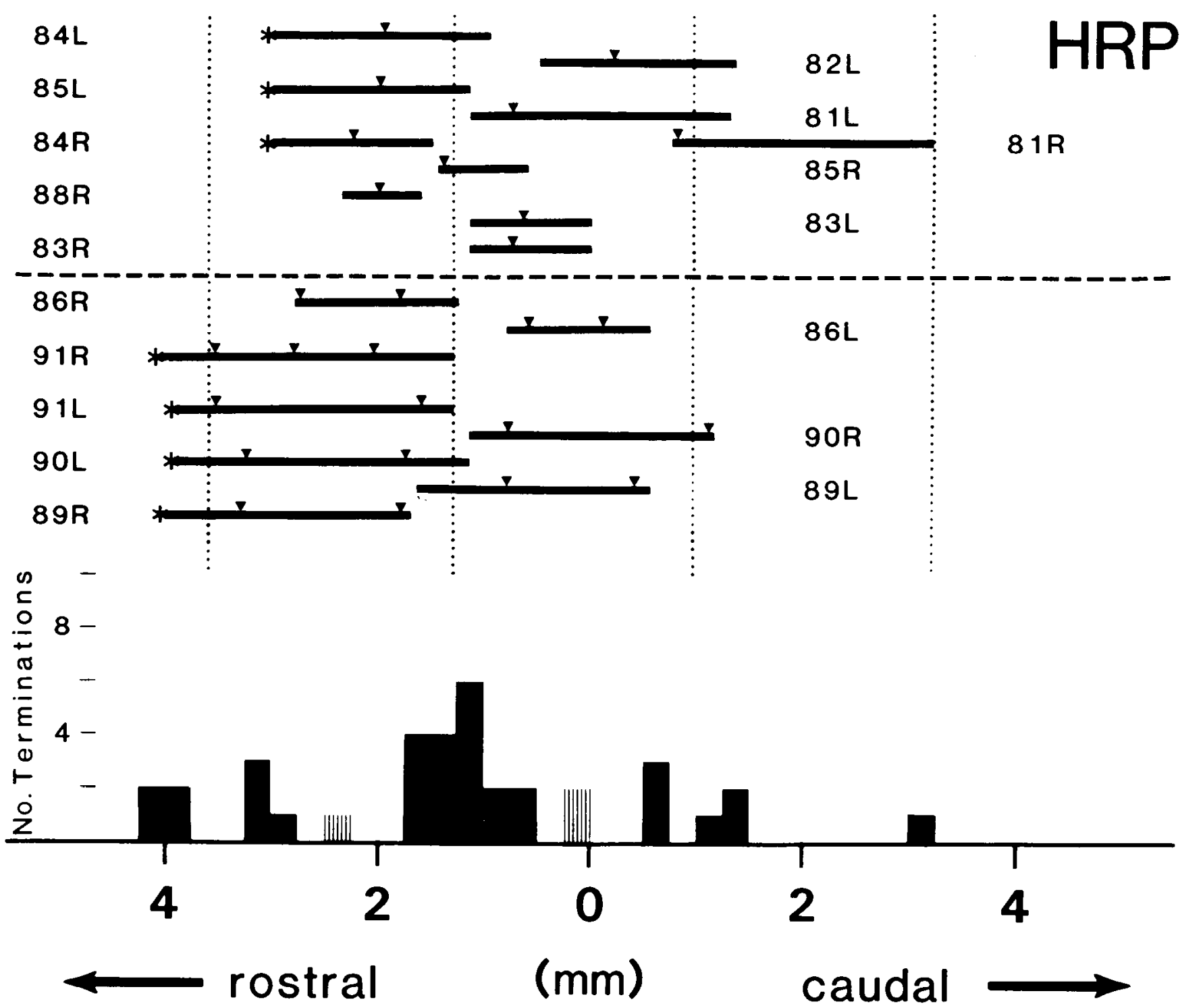

Figure 5. Summary of goldfish Mauthner axon myelin sheath segments stained by intrasheath HRP injections. Segment lengths were determined using serial reconstructions of longitudinal Vibratome sections. Injection sites are indicated with arrowheads. Terminations at the decussation of the Mauthner axons (see Fig. 3) are labeled with asterisks. The horizontal dashed line separates those experiments where either only one injection (above) or two injections (below) were made within a single internode. At the bottom is a histogram of sheath termination sites, showing clusters at separations of 2.2 to $2.3 \mathrm{~mm}$. The vertical dotted lines indicate average termination sites which are, from left to right: $3.6 \mathrm{~mm}$ rostral, $1.3 \mathrm{~mm}$ rostral, $1.0 \mathrm{~mm}$ caudal, and $3.2 \mathrm{~mm}$ caudal to the posterior margin of the vagal lobe $(0 \mathrm{~mm})$. These correspond to the decussation and nodal regions 1,2 , and 3 , respectively. Three terminations (lined boxes in the histogram) were not included in the averages because they were farther than 2 SD from the means and probably represented sheaths that were incompletely stained.

the histogram of Fig. 5) was not included in the averages, as the segments were considered to be only portions of incompletely stained oligodendrocytes.

What is striking about the data summarized in Figures 3 and 5 is that despite the many possible sources of error listed above, the average sites of termination clustered, without overlap, in distinct regions of the brain, and corresponding termination sites are within several hundred micrometers of each other as determined from both the LY and the HRP experiments. Thus, the measurement errors do not appear to have compounded each other. Furthermore, although there is clearly an uncertainty of several hundred micrometers in the overall average sites of termination, such an error is not critical in the context of measuring individual sheath segment lengths of about 2.2 to $2.6 \mathrm{~mm}$.

Previous electrophysiological analyses (Funch and Faber, $1982 \mathrm{~b}$ ) have demonstrated that active site foci are spaced about every $2.4 \mathrm{~mm}$ along the Mauthner axon beginning at the axon hillock-initial segment. Therefore, the myelin sheath segments correspond both in their lengths and in their absolute locations to the regions in between the active site foci. Such correspondence strongly suggests that a node-internode-node structural arrangement exists along the Mauthner axon just as it does along all other myelinated axons which have been studied to date.

Correlation of the spatial sensitivity of the Mauthner axon to TTX with morphological and electrophysiological data. Extracellular ejections of TTX just outside the Mauthner fiber demonstrated that rapid blockage of action potential components only occurred when the ejections were made in regions of the CNS corresponding to the average sites of termination of the oligodendrocytes. These blockages were all-or-nothing events that occurred within seconds, and so they could not have resulted from diffusion of 'TTX over an extended region 


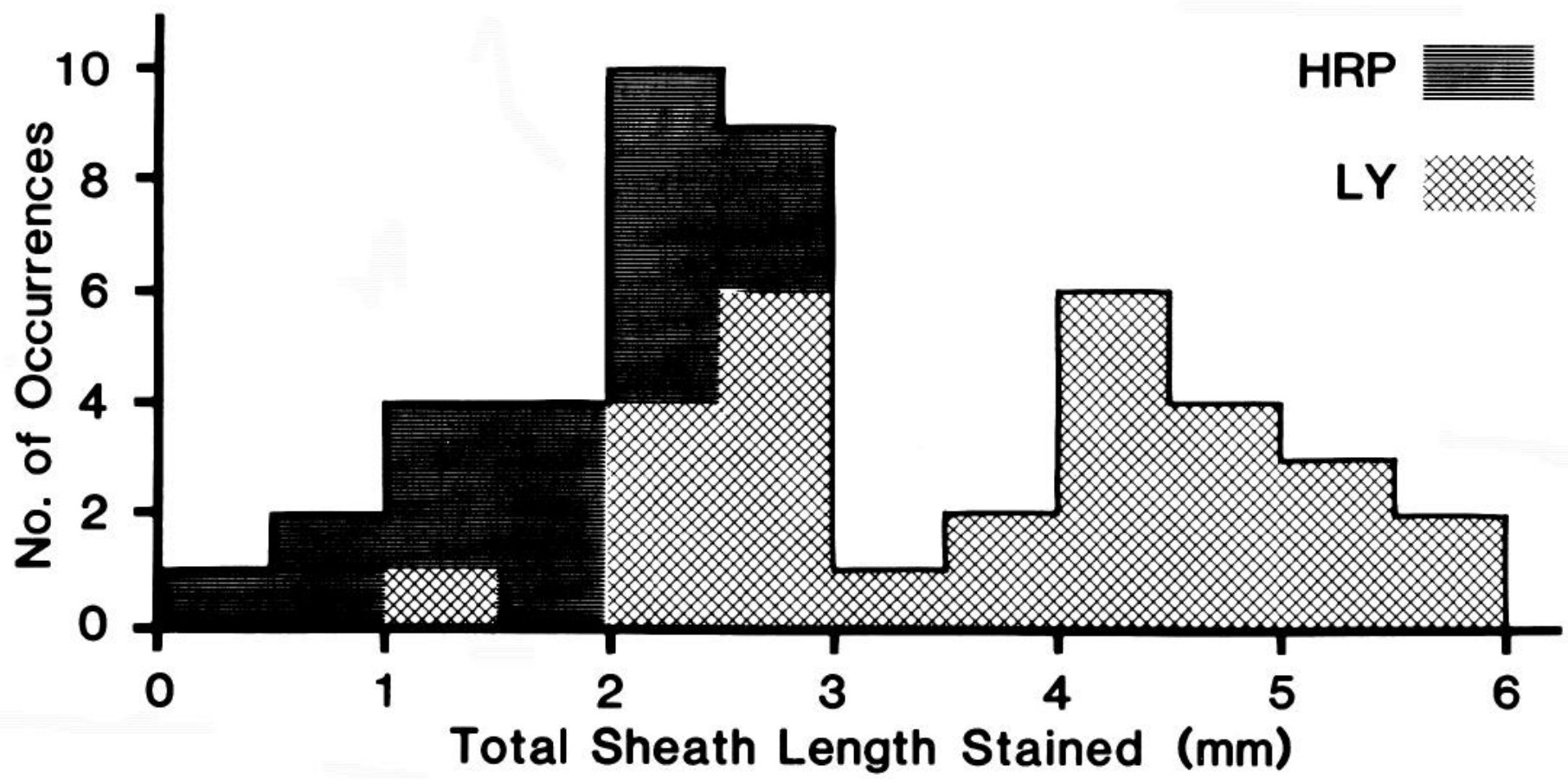

Figure 6. Histogram of myelin sheath segment lengths stained with both HRP and LY. This graph includes all data from Figures 3 and 5 , except that below the thin dashed line in Figure 3. A bimodal distribution is apparent $\left(m_{1}=2.0\right.$ to $2.5 \mathrm{~mm}, m_{2}=4.0$ to $\left.4.5 \mathrm{~mm}\right)$. Lengths of HRP-stained sheaths show a unimodal distribution $(m=2.0$ to $2.5 \mathrm{~mm})$, whereas LY generally stained either short (2 to $3 \mathrm{~mm})$ or longer ( 4 to $6 \mathrm{~mm}$ ) segments and only a few of intermediate length (3 to $4 \mathrm{~mm})$.
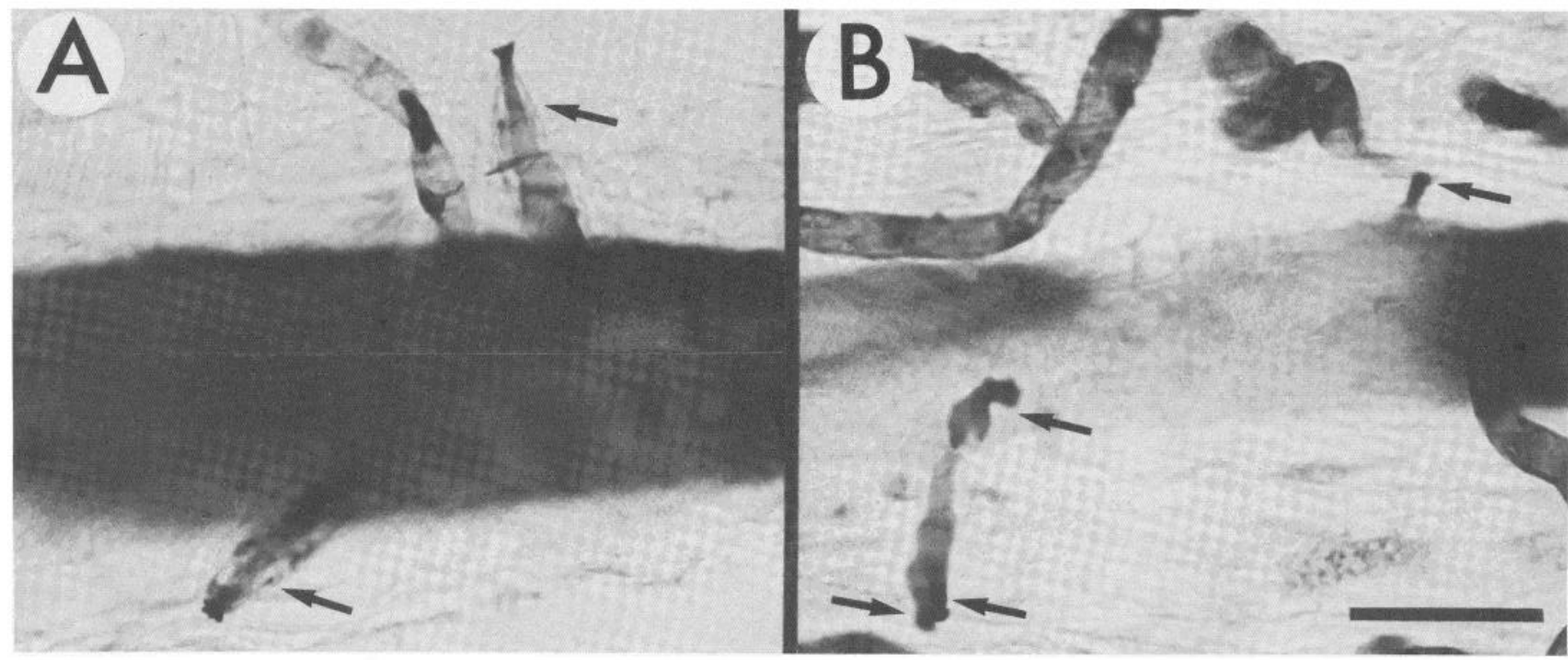

Figure 7. Mauthner axon collaterals revealed by intra-axonal HRP injections. $A$ and $B$ show examples from two different Mauthner axons. In the montage of $A$, two collaterals (arrows) emerge from the axon at different focal planes. In both instances, the individual collateral is ensheathed in myelin with only the tip being free of myelin; this is the region where synapses are located. A blood vessel is stained to the left of the upper collateral. In $B$, four collaterals (arrows) are visible, of which three are clustered together. Due to the angle of sectioning, the bottom two collaterals are nearly superimposed on top of each other, but they could be resolved by focusing through the section. Several blood vessels are evident. The Mauthner axon sheath was not stained and is not visible in either of these micrographs. Calibration line is $50 \mu \mathrm{m}$ for $A$ and $B$.

of the Mauthner axon. This fact was also confirmed by visual monitoring of the spread of the fast green-stained TTX solution. The all-or-nothing antidromic action potential components lost were significant. With an intra-axonal recording site midway between the first and second average sites of termination, the average reduction resulting from a single TTX ejection at the first site was $11 \%$, to $95 \mathrm{mV}$, from a peak control amplitude of $107( \pm 10.4 \mathrm{mV}$ ) (see Table I). Previous analyses 


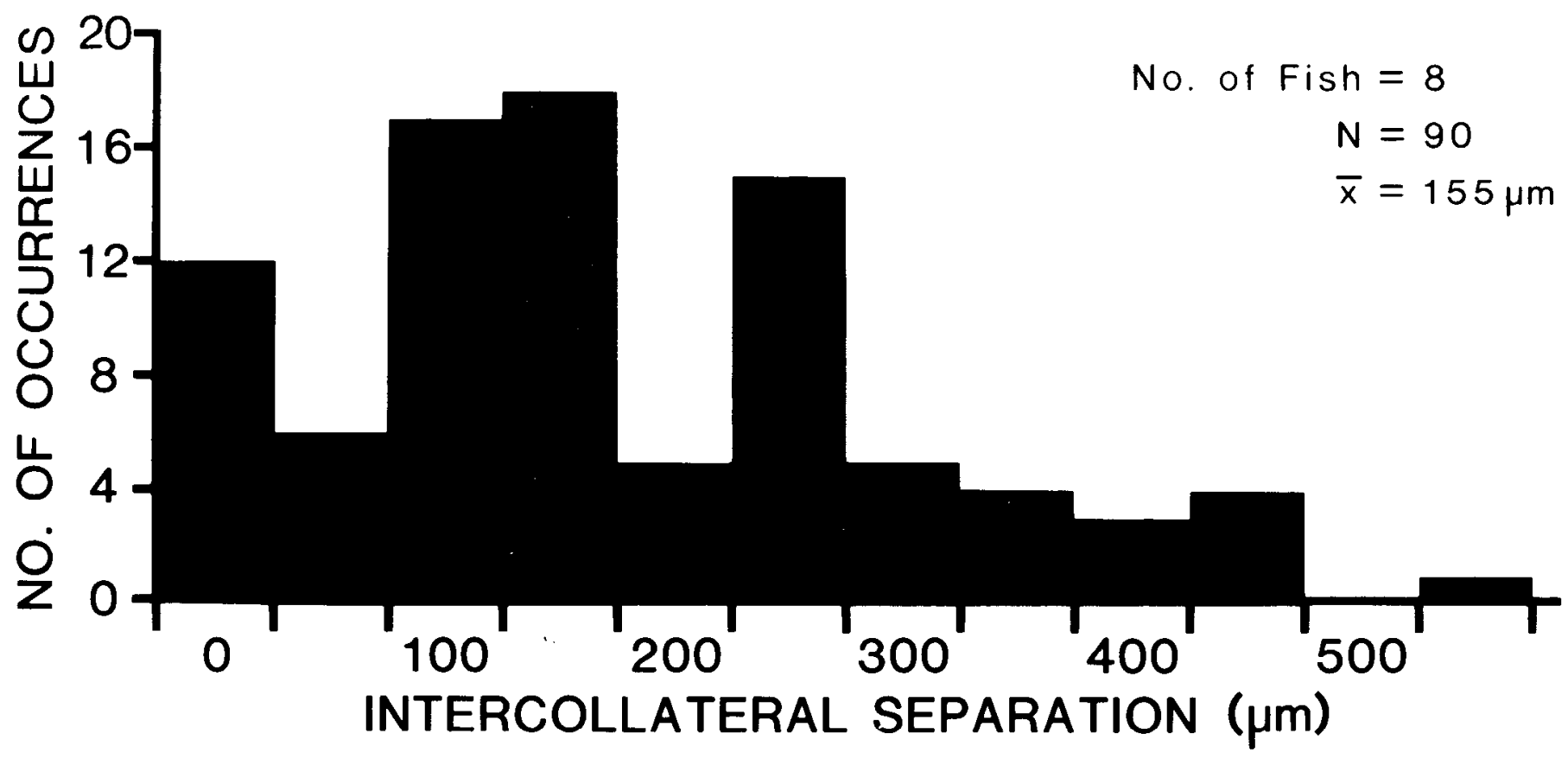

Figure 8. Histogram of distances between Mauthner axon collaterals. For eight fish in which at least one Mauthner axon was injected with HRP, a total of 140 collaterals were observed in 41 sections, each of which contained at least two collaterals. The distances between nearest neighbors within a section were measured, and a total $(N)$ of 90 such intercollateral separations was obtained. The average separation calculated from this histogram is $155 \mu \mathrm{m}$ and is comparable to the average intercollateral distance of $168 \mu \mathrm{m}$ determined from the total number of collaterals (64) observed along $10.74 \mathrm{~mm}$ of two of these axons. Thus, the former sampling technique, which would tend to miss longer separations, introduced little bias. The 12 cases of zero separation were paired and triplet collaterals, arising either from the same point on the axon or as branches of a single stem.

CONTROL

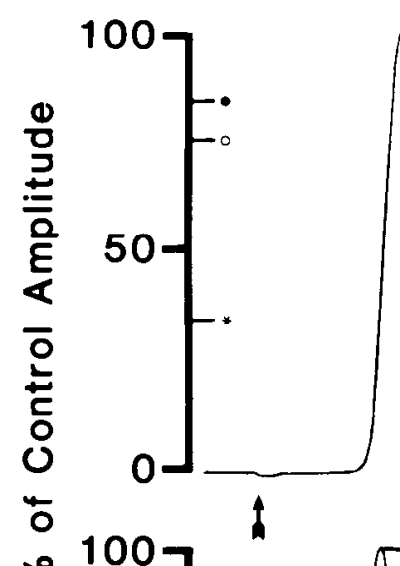

1
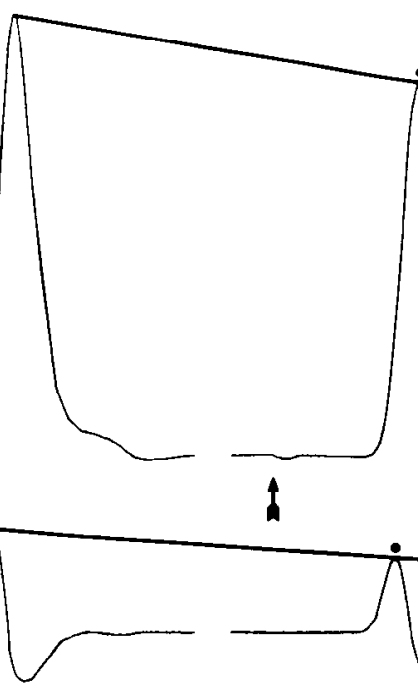

2

3

\&
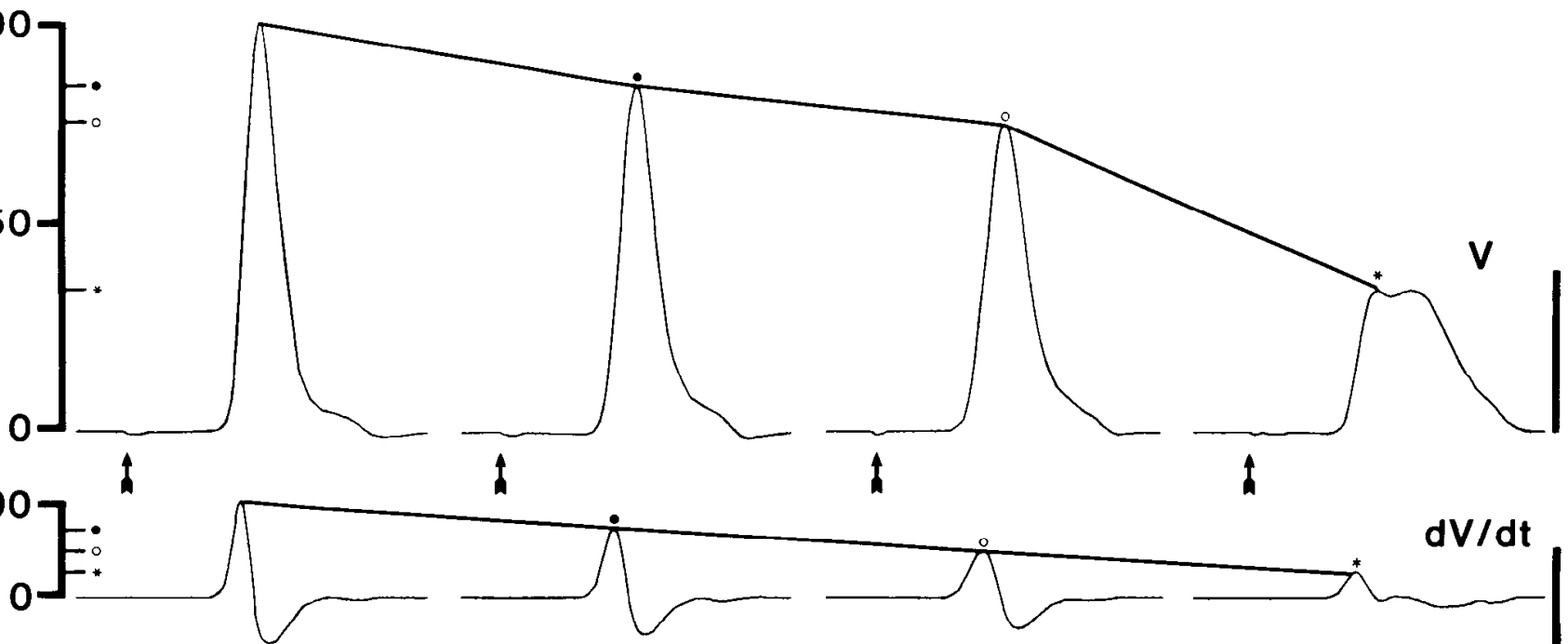

$d V / d t$

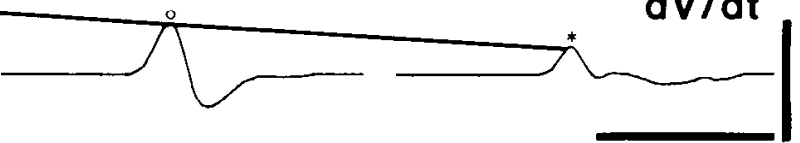

Figure 9. Effects of TTX applied to regions of the Mauthner axon where stained myclin sheath segments terminated. Successive, localized ejections of TTX from a microelectrode in the regions $1.4 \mathrm{~mm}$ rostral $(1,0), 1.2 \mathrm{~mm}$ caudal $(2,0)$, and then $3.2 \mathrm{~mm}$ caudal $(3, *)$ to the posterior margin of the vagal lobes resulted in rapid, all-or-nothing decrements in antidromic action potential amplitude $(V)$ and its digitally differentiated record $(d V / d l)$, as recorded at the posterior margin at the vagal lobe. The unequal percentage decrements in $V$ (relative to control amplitude) are consistent with the passive and active parameters of the Mauthner axon, while the nearly equal percentage decrements in maximal $d V / d t$ are consistent with the correlation between that parameter and the maximal sodium conductance "seen" by the microelectrode (see the text). Arrows indicate stimulus artifacts. Records have been traced by hand from dot matrix printer output. Calibrations are $20 \mathrm{mV}, 1000 \mathrm{~V} / \mathrm{sec}$, and 1 msec.

have determined that the safety factor for impulse propagation along the Mauthner axon is probably at least 6, rheobasic spike threshold is at least $14 \mathrm{mV}$, and axonal space constant is about $5 \mathrm{~mm}$ (Funch and Faber, 1982b). These data can be used to predict the effects on action potential amplitude and its maximal rate of rise as one or more nodes are blocked (see values in parentheses in Table I). Specifically, if a single active site (node 1) is completely blocked by TTX, the action current produced 
TABLE I

All-or-nothing losses in antidromic spike amplitude and its maximal rate of rise resulting from localized $T T X$ ejections in regions where myelin sheath segments terminate.

\begin{tabular}{ccc}
\hline \multirow{2}{*}{$\begin{array}{c}\text { No. of Consecutive } \\
\text { Nodes Blocked by TTX }\end{array}$} & \multicolumn{2}{c}{ Decrement from Control Amplitude } \\
\cline { 2 - 3 } & $11 \pm 2.8 \%^{b}$ & $21 \pm 9.1 \%$ \\
\hline 1 & $(11 \%)^{c}$ & $(25 \%)$ \\
2 & $30 \pm 6.8 \%$ & $45 \pm 14.8 \%$ \\
& $(38 \%)$ & $(50 \%)$ \\
3 & $58 \pm 4.9 \%$ & $66 \pm 7.3 \%$ \\
& $(63 \%)$ & $(65 \%)$ \\
\hline
\end{tabular}

${ }^{a}$ Control spike amplitude $(V)=107 \pm 10.4 \mathrm{mV}$. Control maximal rate of rise of spike $(d V / d t)=957 \pm 124 \mathrm{~V} / \mathrm{sec}$.

${ }^{b}$ Experimental values are mean $\pm \mathrm{SD}, n=8$.

${ }^{c}$ Values in parentheses are theoretical predictions, based upon a safety factor for impulse propagation of 6 , a rheobasic impulse threshold of $14 \mathrm{mV}$, and an internodal distance of one-half the axonal space constant (see the text for details).

by the upstream (caudal) active sites will passively depolarize the inactive site by at least $6(14)=84 \mathrm{mV}$. Thus, the potential at the actual recording site is predicted to be between 84 and $107 \mathrm{mV}$, which is similar to what was observed. When a second antidromic action potential component was blocked with a second TTX ejection at the next caudal (Fig. 9, trace 2) average termination site, the average amplitude which resulted was $30 \%$ less than control, or $75 \mathrm{mV}$. Again, the action current reaching the most caudal blocked active site region (node 2) is 6 times rheobasic current threshold, and $78 \%$ of that current will reach a microelectrode located midway between the blocked sites (nodes 1 and 2) which are about one-half space constant $(\sim 2.5 \mathrm{~mm})$ away from each other; thus, a passive depolarization of at least $0.78(84)=66 \mathrm{mV}$ will be produced at the recording site. Again, this prediction is close to what was observed. Finally, when a third action potential component was blocked, the average amplitude of the remaining impulse was $58 \%$ of control, or $45 \mathrm{mV}$ (Fig. 9, trace 3). Under these conditions, the action current reaching the microelectrode is predicted to be $47 \%$ of that at the most caudal blocked site (node 3 ) which is three-fourths of a space constant away; this would result in a passive depolarization of $0.47(84)=40 \mathrm{mV}$.

The maximal rate of rise of an impulse also yields important information relevant to active site function, as it is proportional to the maximal sodium conductance "seen" by an electrode (Hunter et al., 1975; Walton and Fozzard, 1983a, b). Since the Mauthner axon's active sites are spaced about one-half space constant apart (Funch and Faber, 1982a, b), at least ninetenths of the total conductance "seen" is contributed by the six or seven active sites nearest to the microelectrode. With a recording site midway between two active sites, the predicted contributions to the maximal sodium conductance are approximately $25 \%$ for each of the two active sites on either side of the recording site and $15 \%$ for each of the next distal active sites. Therefore, as the three nearest active sites are successively blocked, the predicted decrements from control maximal rate of rise are $25 \%, 50 \%$, and $65 \%$, respectively. These values compare favorably with the average decrements observed in these experiments (see Table I).

Finally, a safety factor of 6 for impulse propagation means that an impulse can decrement passively over a distance of 2.0 space constants beyond a blocked active site and still be just suprathreshold for impulse initiation and propagation. For the Mauthner axon, this predicts that an impulse should be able to pass through a region where four consecutive active sites are blocked with TTX to activate the next unblocked active site. This was the case when the Mauthner axon was activated orthodromically. Invasion of the axon hillock-initial segment region with antidromic activation failed when the four proximal active sites were blocked by TTX, presumably because of the lower safety factor for antidromic invasion of that region (Funch and Faber, 1982b).

In summary, these experiments demonstrate that the amplitudes of all-or-nothing action potential components that were rapidly blocked by TTX applications in discrete and widely spaced regions of the CNS are consistent with predictions based on the known active and passive (cable) properties of the Mauthner axon and on an active site spacing of about $2.5 \mathrm{~mm}$ (Funch and Faber, 1982a, b). Similar results could not have been obtained if each of the closely spaced collaterals (Figs. 7 and 8 ; average separation $=155 \mu \mathrm{m}$ ) was capable of producing action current for the following reasons: (1) TTX ejections made several hundred micrometers away from the electrophysiologically localized active sites were either ineffective or the resulting component blockage was very delayed (many minutes), indicating that the TTX had to diffuse over a considerable distance in order to block (a) site(s) which contributed a single action potential component. (2) As found in the previous electrophysiological experiments which used axonal hyperpolarization to block axonal spike components, there were only a few, large, all-or-nothing components which could be blocked by TTX. Thus, individual collaterals could not be contributing equally to the production of action current, unless the TTX diffused over a $2.4-\mathrm{mm}$ segment of the axon and blocked all of the randomly spaced collaterals essentially simultaneously. But there is no reasonable explanation for why TTX would diffuse rapidly over the whole axon when ejected at certain locations but not when ejected at other sites. Visually, the rate of diffusion of fast green did not appear different at the various sites along the neuraxis, although a quantitative assessment of this possibility was not performed.

The electrophysiological evidence provided by Greeff and Yasargil (1980) in support of the collateral "nodal equivalent" hypothesis of saltatory impulse propagation along the Mauthner axon has been previously criticized (Funch and Faber, 1982b). Their evidence does not rule out other hypotheses, including the one that holds that distinct active sites are spaced about every $2.4 \mathrm{~mm}$ along the axon. Therefore, no electrophysiological evidence exists that requires the collateral hypothesis for its explanation, whereas all of the electrophysiological and pharmacological results we have obtained (1) are consistent with widely spaced (2.2 to $2.6 \mathrm{~mm}$ ) active sites, (2) can explain Greeff and Yasargil's data, and (3) are incompatible with the hypothesis that all axon collaterals contribute to action current production.

Critique of morphological evidence supporting the collateral "nodal equivalent" hypothesis. The only evidence offered to support the collateral hypothesis which may be incompatible with the hypothesis that sites of action current production are separated by 2.2 to $2.6 \mathrm{~mm}$ is the report of consistent staining patterns observed at all collaterals using various chemical reagents (Yasargil et al., 1982). These include an undercoating of the collateral tip plasmalemma, a reaction product at the collateral tip using the Quick and Waxman (1977) ferric ion/ ferrocyanide staining technique, a reaction product at the collateral tip using a copper thiocholine and ferricyanide (modified Koelle) reagent (Zenker, 1964, 1969; Tsuji, 1974) and the presence of widened and electron-dense extracellular space surrounding the collateral tip (Metuzals, 1965). While such histochemical staining patterns have been observed at sites such as nodes of Ranvier and/or initial segments, they have not yet been correlated specifically with voltage-sensitive sodium (or other ion) channels. For example, the Quick-Waxman technique does not stain unmyelinated axons, and, by using different fixative buffers, it can stain both intracellular and extracellular structures in addition to some membrane-associated 
elements located beneath the axolemma of nodes of Ranvier and initial segments. These findings indicate that the structures so stained are simply associated with regions of high sodium channel density. In fact, preliminary results in our laboratory have demonstrated that the cytoplasm of glial cells (presumably astrocytes) and of some axon terminals is occasionally darkly stained with the Quick-Waxman technique (M. R. Wood, unpublished observations). In addition, the modified Koelle reagent stains regions with high $\mathrm{AChE}$ activity, such as neuromuscular junctions (Zenker, 1964; Tsuji, 1974) and motoneurons (Tsuji, 1975), and Day et al.'(1983) have localized AChE at Mauthner fiber giant synapses in the hatchet fish. Furthermore, the metallophilic reaction product, like the Quick-Waxman one, is localized beneath the nodal axolemma (Zenker, 1969). Likewise, widened and electron-dense extracellular spaces are found not only at nodes of Ranvier but also at CNS and PNS synapses (Smith and Sjöstrand, 1961; Robertson et al., 1963); they have not, to our knowledge, been described at initial segments. Thus, the staining patterns which are observed at the collateral tips are intriguing but cannot be definitively associated with excitable membrane. Certainly, they do not provide strong independent evidence for the collaterals being "nodal equivalents." While we do not rule out the possibility that each of the collaterals possesses some sodium channels, the density of the latter must be so low that most or all collaterals are functionally insignificant with respect to impulse propagation. Clearly, whatever is the active site substrate, it must be readily accessible to the extracellular space. Since the only openings in the myelin sheath observed to date are collateral-like structures, such a protrusion is a likely candidate for the active site substrate. Our evidence simply indicates that the "functional nodes" are fewer in number and spaced more distantly than the collateral distribution can explain. Thus, either the collateral population is heterogeneous with respect to sodium channel density, or else specialized structures resembling collaterals exclusively contain the active membrane.

Summary and significance. The major conclusion from these experiments is that the domains of oligodendrocytes which myelinate the Mauthner axon extend 2.2 to $2.6 \mathrm{~mm}$ between adjacent active sites. Thus, by analogy with all other myelinated axons which have been studied to date, it would seem reasonable to call an active site a node and the region spanned by an oligodendrocyte an internode. The only features which then distinguish the Mauthner axon from other vertebrate myelinated axons are its large size and its lack of typical nodal gaps. However, in regard to this latter characteristic, it should be appreciated that not very long ago many researchers (including Ranvier!) believed that nodes of Ranvier did not exist within the CNS (see discussion in Bunge, 1968), structural features of PNS and CNS nodes may differ (Bunge, 1968; Phillips et al., 1972; Raine, 1977), and even nodes along single vertebrate PNS (Waxman et al., 1972) and CNS (Waxman, 1972) axons may differ considerably. In view of these findings, it seems premature to expect uniformity in the structures of CNS nodes. The observed LY dye coupling may be related to a very close apposition of neighboring oligodendrocytes which might obscure an underlying nodal membrane. In fact, we have never observed any specialization (e.g., constriction or thinning of the myelin sheath, paranodal loops, differential HRP staining, etc.) anywhere along the Mauthner fiber. As stated above, perhaps the nodes are specialized "collaterals" which protrude from the axon. Or the nodes may be small pits in the myelin similar to the dorsal nodes along the earthworm pseudomyelinated median giant fiber (Günther, 1976). Nevertheless, despite the particular nodal morphology, the identifiable Mauthner cell and axon also have functionally identifiable nodes and internodes. This experimental advantage should allow the study of such areas as the sequelae to discrete (e.g., single internodal) demyelinating lesions, the in vivo responses of CNS nodes to locally administered pharmacological compounds, the nature of the Mauthner axon-oligodendrocyte structural relationships over the life span of a fish, and the possibility of intercellular transfer of injected material from axon to oligodendrocyte and vice versa.

\section{References}

Adams, J. C. (1981) Heavy metal intensification of DAB-based HRP reaction product. J. Histochem. Cytochem. 29: 775.

Barets, A. (1961) Contribution a l'étude des systèmes moteurs "lent" et "rapide" du muscle latéral des téléostéens. Arch. Anat. Microsc. Morphol. Exp. 50: 91-187.

Bartelmez, G. W. (1915) Mauthner's cell and the nucleus motorius tegmenti. J. Comp. Neurol. 25: 87-128.

Bodian, D. (1952) Introductory survey of neurons. Cold Spring Harbor Symp. Quant. Biol. 17: 1-13.

Bunge, R. P. (1968) Glial cells and the central myelin sheath. Physiol. Rev. 48: 197-251.

Celio, M. R. (1976) Die Schmidt-Lantermann'schen Einkerbungen der Myelinscheide des Mauthner-axons: orte Longitudinalen Myelinwachstums? Brain Res. 108: 221-235.

Celio, M. R., E. G. Gray, and G. M. Yasargil (1979) Ultrastructure of the Mauthner axon collateral and its synapses in the goldfish spinal cord. J. Neurocytol. 8: 19-29.

Coghill, G. E. (1934) New anatomical relations and the probable function of Mauthner's fibers. Psychiatr. Neurol. 38: 386-391.

Day, J. W., D. H. Hall, L. M. Hall, and M. V. L. Bennett (1983) $\alpha-$ Bungarotoxin labeling and acetylcholinesterase localization at the Mauthner fiber giant synapse in the hatchetfish. J. Neurosci. 3: 272279.

Diamond, J. (1971) The Mauthner cell. In Fish Physiology, W. S. Hoar and D. J. Randall, eds., Vol. V, pp. 265-346, Academic Press, New York.

Diamond, J., E. G. Gray, and G. M. Yasargil (1970) The function of the dendritic spine: An hypothesis. In Excitatory Synaptic Mechanisms, P. Andersen and J. K. S. Jansen, eds., pp. 213-224, Scandinavian University Books, Oslo.

Frank, E., W. A. Harris, and M. B. Kennedy (1980) Lysophosphatidyl choline facilitates labeling of CNS projections with horseradish peroxidase. J. Neurosci. Methods 2: 183-189.

Funch, P. G., and D. S. Faber (1982a) Cable properties of goldfish Mauthner axon. J. Neurophysiol. 47: 1196-1213.

Funch, P. G., and D. S. Faber (1982b) Action-potential propagation and orthodromic impulse initiation in Mauthner axon. J. Neurophysiol. 47: 1214-1231.

Funch, P. G., and D. S. Faber (1983) Pharmacologic evidence for saltatory conduction along the goldfish Mauthner axon. Soc. Neurosci. Abstr. 9: 21.

Graham, H. T., and J. L. O'Leary (1941) Fast central fibers in fish. Properties of Mauthner and Müller fibers of medullospinal system. J. Neurophysiol. 4: 224-242.

Gray, E. G. (1970) The fine structure of nerve. Comp. Biochem. Physiol. 36: 419-448.

Greeff, K. (1976) Impulse conduction in a myelinated giant nerve fibre. Experientia 32: 756 .

Greeff, K. (1977) A method to locate electrically excitable sites along a myelinated giant nerve fibre. Int. Congr. Physiol. Sci. 27th Paris 13: 281.

Greeff, N. G., and G. M. Yasargil (1980) Experimental evidence for saltatory propagation of the Mauthner axon impulse in the tench spinal cord. Brain Res. 193: 47-57.

Günther, J. (1976) Impulse conduction in the myelinated giant fibers of the earthworm. Structure and function of the dorsal nodes in the median giant tiber. J. Comp. Neurol. 168: 505-532.

Hunter, P. J., P. A. McNaughton, and D. Noble (1975) Analytical models of propagation in excitable cells. Prog. Biophys. Mol. Biol. 30: $99-144$.

Koenig, E. (1978) Molecular biology of the Mauthner axon. In Neurobiology of the Mauthner Cell, D. S. Faber and H. Korn, eds., pp. 167182, Raven Press, New York.

Leghissa,'S. (1956) Contribution ultérieure a une meilleure connaissance de l'appareil de Mauthner chez les poissons et observations sur la morphologie de la fibre. In International Meeting of Neurobiolo- 
gists: Progress in Neurobiology, J. Ariens Kappers, ed., pp. 45-62, Elsevier, Amsterdam.

Metuzals, J. (1965) Ultrastructure of the nodes of Ranvier and their surrounding structures in the central nervous system. Z. Zellforsch. 65: 719-759.

Phillips, D. D., R. G. Hibbs, J. P. Ellison, and H. Shapiro (1972) An electron microscopic study of central and peripheral nodes of Ranvier. J. Anat. 111: 229-238.

Quick, D. C., and S. G. Waxman (1977) Specific staining of the axon membrane at nodes of Ranvier with ferric ion and ferrocyanide. J. Neurol. Sci. 31: 1-11.

Raine, C. S. (1977) Morphological aspects of myelin and myelination. In Myelin, P. Morell, ed., pp. 1-49, Plenum Press, New York.

Retzlaff, E., and J. Fontaine (1960) Reciprocal inhibition as indicated by a differential staining reaction. Science 131: 104-105.

Robertson, J. D., T. S. Bodenheimer, and D. E. Stage (1963) The ultrastructure of Mauthner cell synapses and nodes in goldfish brains. J. Cell Biol. 19: 159-199.

Smith, C. A., and F. S. Sjöstrand (1961) Structure of the nerve endings on the external hair cells of the guinea pig cochlea as studied by serial sections. J. Ultrastruct. Res. 5: 523-556.

Stewart, W. W. (1978) Functional connections between cells as revealed by dye-coupling with a highly fluorescent naphthalimide tracer. Cell 14: 741-759.

Thomas, P. K., and J. Z. Young (1949) Internode lengths in the nerves of fishes. J. Anat. 83: 336-350.

Tsuji, S. (1974) On the chemical basis of thiocholine methods for demonstration of acetylcholinesterase activities. Histochemistry 42 :
99-110.

Tsuji, S. (1975) Histochemical demonstration of acetylcholinesteraserich cells in spinal electromotor nucleus of Electrophorus electricus. Brain Res. 88: 499-501.

Walton, M., and H. A. Fozzard (1983a) Experimental study of the conducted action potential in cardiac Purkinje strands. Biophys. J. 44: $1-8$.

Walton, M., and H. A. Fozzard (1983b) The conducted action potential. Models and comparison to experiments. Biophys. J. 44: 9-26.

Waxman, S. G. (1972) Regional differentiation of the axon: A review with special reference to the concept of the multiplex neuron. Brain Res. 47: 269-288.

Waxman, S. G., G. D. Pappas, and M. V. L. Bennett (1972) Morphological correlates of functional differentiation of nodes of Ranvier along single fibers in the neurogenic electric organ of the knifefish Sternarchus. J. Cell Biol. 53: 210-224.

Wood, M. R., P. G. Funch, and D. S. Faber (1983) Identification of internodes in the goldfish Mauthner axon with intra-sheath injection of Lucifer Yellow or HRP. Soc. Neurosci. Abstr. 9: 341.

Yasargil, G. M., N. G. Greeff, H. R. Luescher, K. Akert, and C. Sandri (1982) The structural correlate of saltatory conduction along the Mauthner axon in the tench (Tinca tinca L.): Identification of nodal equivalents at the axon collaterals. J. Comp. Neurol. 212: 417-424.

Zenker, W. (1964) Über die Anfärbung der Ranvierschen Schnürringe beim Koelle-Verfahren zum histochemischen Nachweis der Cholinesterase. Acta Histochem. 19: 67-72.

Zenker, W. (1969) Cholinesterase und Metallophilie an den Ranvierschen Schnürringen. Acta Histochem. 33: 247-273. 